\title{
Síndromes poliglandulares autoinmunes. Diagnóstico y seguimiento en Atención Primaria
}

\author{
I. Prieto Checa, S. Martínez Machuca, V. García Espinosa \\ Espedalistas en Medicina Familiar y Comunitaria. \\ Trabajo actual como médicos de Atención Primaria. Centro de Salud La Ventilla. Madrid
}

\section{RESUMEN}

Los síndromes poliglandulares autoinmunes se diagnostican por la disfunción conjunta de dos o más glándulas asociadas a otras enfermedades no endocrinológicas de etiología autoinmune.

Las disfunciones glandulares son de diagnóstico frecuente en Atención Primaria, siendo necesaria la búsqueda activa de posibles asociaciones para llegar a diagnósticos más precisos en el caso de existir asociaciones.

Un correcto diagnóstico del paciente nos apor ta información válida, tanto para su tratamiento como para realizar el seguimiento familiar, ade cuado en enfermedades con herencia demostrada.

Palabras clave: Síndrome poliglandular autoin mune. Síndrome de Schmidt. Autoinmune.

\section{Polyglandular autoinmune syndromes. Diagno - sis and treatment in Primary Health Care}

\section{ABSTRACT}

Polyglandular Autoimmune Syndrome is diagno sed when two or more endocrine systems are dys functional, along with other non-endocrine immune disorders, specially autoimmune skin diseases.

Glands dysfunctions are often descrived at Pri mary Health Care. After that, it is necessary an ac tive searching of associations to get the diagnosis of a possible syndrome.

A correct diagnosis give as more information, to improve the threatment and start a correct familiar following, specially when it is proven a genetic he redity as in this case.

Key words: Polyglandular autoimmune syndro me. Schmidt syndrome. Autoimmune.

\section{INTRODUCCIÓN}

La aparición de alteraciones de tipo inmunitario asociadas en un mismo paciente debe hacernos pensar en el diagnóstico de síndromes poliglandulares autoinmunes (usaremos las siglas SPA para referirnos a ellos en el texto), especialmente si a las alteraciones funcionales de dos o más glándulas se asocian otros trastornos autoinmunes no endocrinos, siendo los cutáneos los más comunes.

El riesgo de sufrir una segunda patología autoinmune está aumentado en diabetes tipo 1. Por ejemplo, una de cada siete personas con diabetes tipo 1 sufren síndrome poliglandular autoinmune tipo II; un porcentaje suficientemente alto como para justificar la búsqueda de asociaciones en disfunciones endocrinológicas en pacientes con diabetes juvenil.

No es fácil encontrar datos precisos sobre la frecuencia de estos síndromes pues en la mayoría de los casos no se llega al diagnóstico. Las disfunciones glandulares son patologías muy frecuentes en nuestras consultas, por lo que la aparición coincidente de varias de ellas en un mismo paciente no suele sorprendernos.

Es importante reconocer estos síndromes en la práctica clínica y estar alerta ante la posibilidad de un fallo endocrino secundario posterior. El diag- 


\begin{tabular}{|c|c|c|c|}
\hline & $\begin{array}{l}\text { DISFUNCIONES ENDOCRINAS } \\
\text { ASOCIADAS }\end{array}$ & TIPO DE HERENCIA & EDAD DE COMIENZO \\
\hline \multirow[t]{2}{*}{ PGA* tipo I } & $\boldsymbol{V}$ Glándula suprarrenal & $\boldsymbol{V}$ Autosómica recesiva & $\boldsymbol{V}$ Infancia \\
\hline & $\boldsymbol{\sim}$ Glándula paratiroidea & Cromosoma 21q22 & \\
\hline \multirow[t]{3}{*}{ PGA* tipo II } & $\boldsymbol{V}$ Enfermedad de Addison & $\boldsymbol{V}$ Autosómica dominante & $\boldsymbol{\sim}$ Adulta \\
\hline & $\boldsymbol{V}$ Diabetes Mellitus & ligado a HLA DR4 & \\
\hline & $\boldsymbol{\sim}$ Tiroiditis & & \\
\hline \multirow[t]{2}{*}{ PGA* tipo III } & $\boldsymbol{V}$ Glándula tiroidea & $\checkmark$ Autosómica dominante & $\boldsymbol{\sim}$ Adulta \\
\hline & $\boldsymbol{V}$ Diabetes Mellitus & & \\
\hline
\end{tabular}

*Síndrome Poliglandular Autoinmune (PolyGlandular Autoimmune).

nóstico puede ayudarnos en el seguimiento y la información de los pacientes.

Los diagnósticos tempranos previenen significativamente en cuanto a morbilidad y mortalidad por las posibles complicaciones que suceden a lo largo de la vida. El buen conocimiento de estos síndromes facilita que el paciente se involucre en su propio cuidado, al tiempo que conoce mejor la evolución de su enfermedad.

\section{OBSERVACIÓN CLÍNICA}

Se presenta el caso de modo cronológico por ser su modo de aparición más habitual, con largos intervalos entre los diferentes diagnósticos. Es el siguiente:

Mujer de 39 años en el momento actual. Antecedentes familiares destacables de fallecimiento del padre a los cincuenta y dos años de neoplasia pancreática. Madre fallecida con cincuenta y seis a causa de una infección respiratoria, con diagnósticos previos documentados de artritis reumatoidea seronegativa y síndrome de Sjogren. Una tía materna con diagnóstico de síndrome de Sjogren y enfermedad de Raynaud. Diabetes mellitus tipo dos en ambas abuelas.

La paciente es conocida en consulta en 1989 , época en la que se le realizó historia clínica. Hasta ese momento no había tenido ningún ingreso hospitalario ni cirugía. Fumadora de cuarenta cigarrillos al día desde los quince años. Niega consumo de alcohol así como otros consumos tóxicos, y no recibe medicación habitual crónica. La exploración es normal excepto sobrepeso e hipopigmentación cutánea compatible con vitíligo en dedos de manos y región perioral. No recuerda el comienzo del vitíligo, ni refiere antecedentes en su familia. Los estudios en sangre no señalan ninguna anomalía hematológica ni bioquímica que destacar. Se realizan pruebas de hormonas tiroideas encontrándose dentro de límites normales.

En 1992, en las pruebas sanguíneas anuales realizadas en controles laborales se detecta por primera vez hiperglucemia basal, que se confirma al repetir el examen. Los valores de 124 y $121 \mathrm{mg} / \mathrm{dl}$ en dichas pruebas no establecen diagnóstico de diabetes según los criterios actuales. Se le dan pautas dietéticas, insistiendo en el control de peso y en la realización de ejercicio físico frecuente, con buena recepción del consejo por parte de la paciente.

En 1997 los valores basales de glucemia se hacen suficientes para el diagnóstico de diabetes, iniciando terapia con antidiabéticos orales, que en pocos meses ha de ser sustituida por tratamiento con insulina para conseguir control adecuado de glucemias.

En 1999 se detectan por primera vez cifras de tensión arterial altas en controles, llegándose al diagnóstico de hipertensión arterial, de tipo esencial por descartarse otras causas, que no se controla lo suficiente con restricción de sal, pero responde bien al tratamiento diurético, bien tolerado por la paciente.

Unos meses más tarde la paciente acude a nuestra consulta quejándose de cansancio, mala tolerancia al esfuerzo y ánimo decaído. Presenta estos síntomas desde el mes previo. No se acompaña de pérdida de apetito ni de insomnio, y dificulta el control de peso. La exploración de la paciente en 
este caso es similar a la previa. No se observan cambios de coloración en la piel. No refiere edemas, ni signos de sangrado. Los recientes diagnósticos de enfermedades crónicas en una persona joven, nos inducen a pensar en una reacción psicológica a la situación, lo que nos obliga a descartar otras patologías de origen orgánico que pudieran simularla. Los niveles de hemoglobina, así como de hierro vuelven a estar dentro de límites normales. Presenta un buen control glucémico, sin embargo las hormonas tiroideas aparecen alteradas, con TSH cuatro veces por encima de valores normales en los controles hematológicos. Repetida la determinación en una segunda ocasión dos semanas más tarde, se confirma el diagnóstico de hipotiroidismo. Los anticuerpos antiperoxidasa son positivos, mientras que los anticuerpos antimitocondriales así como los antinucleares aparecen negativos.

La buena respuesta clínica al tratamiento con tiroxina confirma el diagnóstico.

Aún con la noticia de su tercera enfermedad crónica la paciente vuelve a estar animada y colaboradora, aunque a veces confiesa que le da un poco de miedo venir al centro de salud: "a ver qué me encuentran la próxima vez".

Actualmente, año 2001, la paciente sigue tratamiento con dieta diabética, insulina, tiroxina y diurético. Su rendimiento laboral es normal, no presenta rasgos depresivos, y califica de buena su calidad de vida.

La asociación de vitíligo, hipotiroidismo y diabetes mellitus está descrita en el síndrome poliglandular autoinmune tipo III. Se llega al diagnóstico por la asociación de disfunción tiroidea autoinmune asociada a cualquier patología autoinmune excluyendo la de glándula suprarrenal. Refuerzan el diagnóstico la coincidencia de vitíligo y la edad de aparición.

Se hace diagnóstico de síndrome poliglandular autoinmune tipo III en la paciente, iniciándose estudio familiar, y dotándola de información precisa de su enfermedad, estableciendo tests periódicos de búsqueda para la prevención de futuras disfunciones endocrinas secundarias.

\section{DISCUSIÓN. SÍNDROMES POLIGLANDULARES AUTOINMUNES}

Son un grupo de síndromes que tienen en común ser la asociación de dos o más enfermedades del sistema endocrino asociadas a otras patologías de causa autoinmune. Generalmente se trata de hipofunciones glandulares, excepto en el caso del tiroides, en que pueden aparecer tanto como hipo como hiperfunción. Se han clasificado en tres grupos: tipo I, II y III, comprendiendo cada uno de ellos asociaciones, herencia y modo de presentación típicos.
La clasificación fue descrita por Neufeld ${ }^{1}$, Blizzard y Maclaren, pero desde el momento de la clasificación ha habido avances importantes en el conocimiento de estos síndromes.

-El síndrome tipo I comienza generalmente durante el primer decenio de vida, en la edad pediátrica. También se la conoce como forma juvenil de SPA. Para el diagnóstico son suficientes dos de los componentes de la triada siguiente: candidiasis mucocutánea, hipoparatiroidismo e insuficiencia suprarrenal. Pueden asociarse otros defectos endocrinos como hipogonadismo o hipotiroidismo. Más rara es la aparición de diabetes. Manifestaciones adicionales como vitíligo, distrofia ungueal, queratopatía, anemia perniciosa o hipoplasia del esmalte dental pueden acompañar. El número de sístemas afectados aumenta con el tiempo. Es necesario el seguimiento a largo plazo ya que ciertos componentes endocrinos pueden no aparecer hasta la cuarta década de la vida. La herencia es de tipo autosómico recesivo ligada a la mutación de un único gen situado en el cromosoma 21. Al gen mutado se le ha llamado AIRE $^{2-4}$ (regulador autoinmune), y presenta una penetrancia del $100 \%$, sin haberse encontrado asociaciones con HLA en cuanto a herencia. Es característica una alta variabilidad en el número y presentación de los componentes del síndrome, lo que sugiere factores ambientales que modulan la aparición de la enfermedad.

—El síndrome tipo II o de Schmidt ${ }^{5-7}$ presenta según su descripción diabetes mellitus, insuficiencia suprarrenal y tiroiditis. Para su diagnóstico son precisas la coexistencia de disfunción corticoadrenal con diabetes o con hipertiroidismo. De inicio en la edad adulta. La presencia de anticuerpos positivos antimicrosomales o antitiroglobulina no predicen la futura aparición de enfermedad tiroidea, que suele presentarse en forma de tiroiditis de Hashimoto. Son menos frecuentes que en el tipo I las asociaciones dermatológicas como vitíligo o alopecia. Hasta en un cuarto de los pacientes diagnosticados de Miastenia Gravis y en una porción superior si ésta se asocia a timona, presentan síndrome poliglandular autoinmune tipo II. Algunos pacientes presentan también hipoparatiroidismo, habitualmente transitorio. La herencia es de tipo autosómico, aunque parece ligada a más de un gen. Se ha encontrado asociación con HLA DR3. Es la forma más frecuente de estos tres síndromes, encontrándose en un estudio ${ }^{8}$ que la prevalencia era del $1 \%$ entre los pacientes de una clínica de endocrinología. Se hace obligado ante el diagnóstico de enfermedad de Addison los controles posteriores del paciente para el posible diagnóstico precoz de las patologías asociadas. Con frecuencia asocia an- 
ticuerpos anticélulas parietales positivos, habiendo sido mencionados como posible marcador.

-El síndrome tipo III es heterogéneo. Consta de enfermedad tiroidea inmunitaria junto con cualquier disfunción glandular excluyendo glándula suprarrenal ${ }^{9}$. De comienzo en la edad adulta. Su más frecuente asociación es a disfunción de los islotes pancreáticos, con la aparición de diabetes. Es el que se asocia con más frecuencia a enfermedades reumatológicas de tipo autoinmune, aunque no es rara su coexistencia con trastornos autoinmunes de tipo cutáneo como la alopecia o el vitíligo, o de la anemia perniciosa. Su tipo de herencia está mal definida. Parece ser de tipo dominante, con alta penetrancia, similar al de tipo II. También ha sido asociada a HLADR3. En diferentes estudios se apunta su asociación a enfermedad celíaca o sarcoidosis.

Es común en las tres formas la herencia autosómica, con importante influencia ambiental con mucha variabilidad en su expresividad como demuestran los estudios de gemelos.

El largo intervalo que sucede entre la aparición de las diferentes enfermedades de tipo endocrino justifica la monitorización regular de los pacientes con enfermedades endocrinas de tipo autoinmune ${ }^{10}$. No está tan clara la conveniencia de realizar test adicionales ante diagnósticos de enfermedades autoinmunes no endocrinas.

El diagnóstico en familiares se realiza con determinaciones de glucemia en ayunas en casos de diabetes, prueba de estimulación de cosintropina en insuficiencia suprarrenal, determinación de TSH, de hormona luteinizante $(\mathrm{LH})$ y foliculoestimulante (FSH) y concentraciones de testosterona en varones. Si el diagnóstico en la familia es de un probable tipo I, debemos añadir la detección de calcio y fósforo. El seguimiento de familiares de primer grado comprende las determinaciones descritas previamente, cada uno o dos años hasta los 50 años en familiares de los tipos II y III, y hasta los 40 si se trata del síndrome tipo I.

El tratamiento es el de las disfunciones endocrinas presentes. Se han ensayado terapias inmunosupresoras ${ }^{11}$ con buenos resultados en algunos casos. El futuro parece estar en la terapia génica, especialmente en el síndrome tipo I, en que el gen mutado es bien conocido, y ha sido totalmente codificado en la actualidad.

\section{CONCLUSIÓN}

Es en el medio de Atención Primaria donde se diagnostican con más frecuencia las disfunciones endocrinas. El largo intervalo que sucede entre la aparición de las diferentes patologías de tipo endocrino justifica la monitorización regular de los pacientes con enfermedades autoinmunes. Sería conveniente contar con estudios de coste -beneficio y coste- oportunidad que avalasen la realización de pruebas periódicas de despistaje ante cualquier disfunción del sistema endocrino de esta etiología, entre las que podrían incluirse pruebas tan accesibles a nuestro medio de trabajo como son determinación de glucemia en ayunas, hormonas tiroideas, e iones en sangre. Valorando la necesidad de añadir las determinaciones de corticotropina, hormonas sexuales.

Conocer las diferentes asociaciones de disfunciones glandulares autoinmunes nos habilita para llegar a un diagnóstico más preciso. Si éste es el adecuado nos permite un mejor tratamiento, seguimiento y asesoramiento al paciente.

Además en síndromes con herencia conocida como este caso nos ayuda a la detección precoz de los casos familiares así como al consejo genético.

La mejor información y conocimiento de su propia patología que el usuario recibe, colabora sin duda a su mayor implicación en su propio seguimiento.

En este caso concreto, resultan curiosos los antecedentes familiares de diferentes enfermedades de etiología autoinmune que la paciente aporta documentados por informes médicos, no correspondiendo en ningún caso a los mencionados síndromes, pero que como ya está descrito parecen dotar de cierta predisposición familiar, no concretando cuál es su modo de herencia.

En los dos hijos de nuestra paciente, de quince y trece años respectivamente, coincide la presentación de vitíligo, sin hallarse ninguna otra anomalía analítica ni hormonal en los últimos controles.

\section{CORRESPONDENCIA:}

Isabel Prieto Checa

Centro de Salud La Ventilla

Avenida de Asturias $n^{\circ} 63$

28029 Madrid 


\section{Bibliografía}

1. Neufeld M, Maclaren NK, Blizzard RM. Two types of autoimmune Addison's disease associated with different polyglandular autoimmune (PGA) syndromes. Medicine (Baltimore) $1981 \mathrm{Sep}$; 60(5): 355-62.

2. Halonen M, Pelto-Huikko M, Eskelin P, Peltonen L, Ulmanen I, Kolmer M. Subcellular location and expression of autoimmune regulator (Aire), the mouse ortologue for human gene defective in autoimmune polyendocrinopathy candidiasis ectodermal dystrophy (APECED). J Histochem Cytochem 2001 Feb; 49(2): 197-208.

3. Shi JD, Wang CY, Marron MP, Ruan QG, Huang YQ, Detter JC, et al. Chromosomal localization and complete genomic sequence of the murine autoimmune regulator gene (Aire). Autoimmunity. 1999; 31(1): 47-53.

4. Chen QY, Lan MS, She JX, Maclaren NK. The gen responsible for autoimmune polyglandular syndrome type I maps to chromosome 21q22.3 in US patients. J Autoimmun 1998 Apr; 11(2): 177-83.

5. Betterle C, Volpato M, Greggio AN, Presotto I. Type 2 polyglandular autoimmune disease (Schmidt's syndrome). J Pediatr Endocrinol Metab 1996 Mar; 9 suppl 1: 113-23.
6. Soriano RJ, Carrera GE, Sánchez PV. Síndrome poliglandular autoinmune tipo II (síndrome de Schmidt). Informe del primer caso diagnosticado en 29,568 autopsias practicadas en el Hospital General de México. Rev Med Hosp Gen Mex 1999; 62(2): 128-31.

7. Takedo R, Takayama Y, Tagawa S, Kornel L. Schmidt's syndrome: autoimmune polyglandular disease of adrenal and thyroid glands. Isr Med Assoc J 1999 Dec; 1(4): 285-6.

8. De Luis DA, Varela C, Lahera M, Sancho Rof J. Prevalencia del Síndrome Poliglandular Autoinmune tipo II en la enfermedad de Addison de origen idiopático. Med Clin (Bar) 1999 May 8; 112(16): 638.

9. Rodriguez- Quiroz F, Renato Berrón PR, Ortega-Martell JA, Onuma-Takane E. Síndrome Poliglandular Autoinmune tipo III. Informe de un caso. Alergia e Inmunol Pediatr 1995; 4(2): 65-7.

10. Obermayer-Straub P, Strasburg CP, Manns MP. Autoimmune hepatitis. J Hepatol 2000; 32 (1suppl): 181-97.

11. Patakfalin A. Cyclosporin A therapy in autoimmune diseases. Orv Hetil 1996 Sep 1; 137(35): 1921-7. 